Draft VERsion February 9, 2014

Preprint typeset using $\mathrm{AT}_{\mathrm{E} X} \mathrm{X}$ style emulateapj v. 08/22/09

\title{
THE DISTRIBUTION OF RECOIL VELOCITIES FROM MERGING BLACK HOLES
}

\author{
Jeremy D. Schnittman and Alessandra Buonanno \\ Department of Physics, University of Maryland, College Park, MD 20742 \\ Draft version February 9, 2014
}

\begin{abstract}
We calculate the linear momentum flux from merging black holes (BHs) with arbitrary masses and spin orientations, using the effective-one-body (EOB) model. This model includes an analytic description of the inspiral phase, a short merger, and a superposition of exponentially damped quasinormal ringdown modes of a Kerr $\mathrm{BH}$. By varying the matching point between inspiral and ringdown, we can estimate the systematic errors generated with this method. Within these confidence limits, we find close agreement with previously reported results from numerical relativity. Using a Monte Carlo implementation of the EOB model, we are able to sample a large volume of BH parameter space and estimate the distribution of recoil velocities. For a range of mass ratios $1 \leq m_{1} / m_{2} \leq 10$, spin magnitudes of $a_{1,2}=0.9$, and uniform random spin orientations, we find that a fraction $f_{500}=$ $0.12_{-0.05}^{+0.06}$ of binaries have recoil velocities greater than $500 \mathrm{~km} / \mathrm{s}$ and $f_{1000}=0.027_{-0.014}^{+0.021}$ have kicks greater than $1000 \mathrm{~km} / \mathrm{s}$. These velocities likely are capable of ejecting the final $\mathrm{BH}$ from its host galaxy. Limiting the sample to comparable-mass binaries with $m_{1} / m_{2} \leq 4$, the typical kicks are even larger, with $f_{500}=0.31_{-0.12}^{+0.13}$ and $f_{1000}=0.079_{-0.042}^{+0.062}$.

Subject headings: black hole physics - relativity - gravitational waves - galaxies: nuclei
\end{abstract}

\section{INTRODUCTION}

In the past year there has been remarkable progress made in the field of numerical relativity (NR). One of the most exciting new results is the calculation of the linear momentum flux generated by the inspiral, merger, and ringdown of black hole $(\mathrm{BH})$ binaries (Herrmann et al. 2006; Baker et al. 2006; Gonzalez et al. 2006; Herrmann et al. 2007; Koppitz et al. 2007; Campanelli et al. 2007; Gonzalez et al. 2007; Baker et al. 2007). Since the majority of this momentum flux is emitted during the merger and ringdown, it is difficult to make definitive predictions for the recoil using only analytic methods. However, in the non-spinning case, the postNewtonian (PN) model (Blanchet et al. 2005) has provided results consistent with $\mathrm{NR}$ all along the adiabatic inspiral; the effective-one-body (EOB) model can reproduce the total recoil, i.e., also the contribution from the ring-down phase, but with large uncertainties (Damour \& Gopakumar 2006); perturbative models (Sopuerta et al. 2006) have also spanned the NR predictions. The recoil remains an ideal problem for which one can benefit enormously from accurate numerical simulations.

Recent NR results predicting very large recoil velocities ( $\gtrsim 500 \mathrm{~km} / \mathrm{s}$ ) have also called attention to the potential astrophysical importance of the recoil (Campanelli et al. 2007; Gonzalez et al. 2007). For many models of dark matter halo growth through hierarchical mergers, supermassive BHs at the centers of such halos will inevitably also merge unless kicked out of the gravitational potential well from a previous merger (Menou et al. 2001). The NR results have shown that this is indeed possible, but have not yet shown whether it is probable or not.

As an initial investigation of this question, we have applied the EOB approach (Buonanno \& Damour 1999, 2000; Damour et al. 2000) to model the BH inspiral and ringdown phases including spin-orbit and spin-spin effects (Buonanno et al. 2006a), described below in $\S 2$. Unlike previous analytic approaches, we can now benefit from a rapidly growing collection of numerical data, allowing us to calibrate our model and quantify its uncertainties. In $\S 3$, we compare a large number of these NR simulations to our EOB predictions, agreeing within our conservative error estimates. Having established a range of confidence in the EOB model, we proceed in $\S 4$ to show the results of Monte Carlo simulations with a wide range of mass ratios, spin magnitudes, and orientations. We thus provide the first estimates of the distribution of recoil velocities from $\mathrm{BH}$ mergers, and in $\S 5$ briefly discuss the astrophysical implications of these results.

\section{ANALYTIC MODEL OF BLACK HOLE RECOIL}

For the two-body dynamics, we use the EOB model with spin-orbit and spin-spin terms included through $1.5 \mathrm{PN}$ and $2 \mathrm{PN}$ order, respectively, as described in Buonanno et al. (2006a). The non-spinning, conservative dynamics are computed through $3 \mathrm{PN}$ order (Damour et al. 2000) and the radiation-reaction effects are included through 3.5PN order (Blanchet et al. 2004). The initial conditions are taken to replicate an adiabatic, quasi-circular inspiral beginning at $r=10 \mathrm{~m}$. Building on previous works (Buonanno \& Damour 2000; Buonanno et al. 2006a; Damour \& Gopakumar 2006), Buonanno et al. (2006b) were able to match the EOB inspiral-plunge waveform to a linear combination of three Kerr quasi-normal ringdown (RD) modes, obtaining qualitative agreement with the full NR inspiral-merger$\mathrm{RD}$ wave. In the model used here, we assume the final $\mathrm{BH}$ spin is given by the linear scaling with mass ratio of Gonzalez et al. (2006). After calculating the inspiral and RD dynamics, we determine the linear momentum flux using the radiative multipole moments described in Thorne (1980), including the leading-order 
radial velocity and spin-orbit contributions to the individual modes (Kidder 1995). These methods will be described in greater detail in a companion paper.

The instantaneous transition from inspiral to ringdown turns out to be rather sensitive to the point of matching and it is only partially effective: the gravitational wave (GW) frequency resulting from the EOB matching grows too quickly during the merger-RD transition, whereas the NR GW frequency increases more gently. As a way of estimating the optimal match point as well as the errors associated with this "prompt merger" approximation, we calculate the GW energy emitted in the inspiral relative to that of the ringdown. Since the amplitudes of the individual multipole modes typically increase rapidly with frequency during the inspiral, by matching to the ringdown at a later time, the amplitudes of the excited RD modes and thus the RD energy increases significantly. Similarly, if we match too early, the resulting RD amplitudes are too small. Following Flanagan \& Hughes (1998), we scale the RD energy according to $E_{\mathrm{RD}}(\mu) \propto \mu^{2} / m$ (we define $m=m_{1}+m_{2}$, $\mu=m_{1} m_{2} / m$, and $\eta=\mu / m$, with $\left.m_{1}>m_{2}\right)$ and scale the inspiral energy to $E_{\text {ins }}(\mu) \propto \mu \varepsilon$, where $1-\varepsilon$ is the specific energy of a test particle at the inner-most stable circular orbit (determined here via the effective spin of the EOB model). Guided by NR results, we set $E_{\text {ins }}=E_{\mathrm{RD}}$ in the equal-mass case (Buonanno et al. 2006b), and use the scaling relations to determine the "target" inspiral and $\mathrm{RD}$ fractions for other $\mathrm{BH}$ mass ratios and spins.

We then vary the matching point in time, calculating the recoil for each case, and requiring the fraction $E_{\text {ins }} /\left(E_{\text {ins }}+E_{\mathrm{RD}}\right)$ to agree with the target fraction within $\pm 15 \%$. This typically corresponds to a range of $r / m \approx 2.5-3.5$ and $\omega_{\text {orb }} \approx 0.1-0.2$ at the matching point. For our EOB recoil predictions, we take the mean kick velocity over the acceptable match range and define 1- $\sigma$ errors from the variance over this range. By calibrating with the NR results and quantifying its uncertainties, the current EOB model can already be used to obtain interesting predictions of the recoil velocity.

\section{COMPARISON WITH NUMERICAL SIMULATIONS}

Unlike previous analytic calculations of the recoil, we are now in the unique position of being able to compare our EOB predictions directly with a growing collection of high-resolution NR simulations and thereby establish a range of confidence in the results of our analytic model. Beginning with the simplest case, in Figure 1 we show in the top panel the EOB recoil velocities for non-spinning unequal-mass binaries (diamond symbols with 1- $\sigma$ error bars), along with the classic formula of Fitchett (1983): $v(\eta) \propto \eta^{2} \sqrt{1-4 \eta}$, scaled to the results of Gonzalez et al. (2006) who find $v\left(\eta_{\max }\right)=175 \mathrm{~km} / \mathrm{s}$. In the bottom panel, we show the results of equal-mass binaries with equal-magnitude spins aligned and anti-aligned with the orbital angular momentum. The solid line is the linear scaling $v(a)=475 a \mathrm{~km} / \mathrm{s}$ given by Herrmann et al. (2007), where we define the dimensionless spin parameter $a_{1,2} \equiv\left|S_{1,2}\right| / m_{1,2}^{2}$.

In Figure 2 we show the combination of the unequalmass and anti-aligned spin effects, with both black holes having equal dimensionless spin magnitudes $a$. Following the approach of Baker et al. (2007), we plot analytic fits
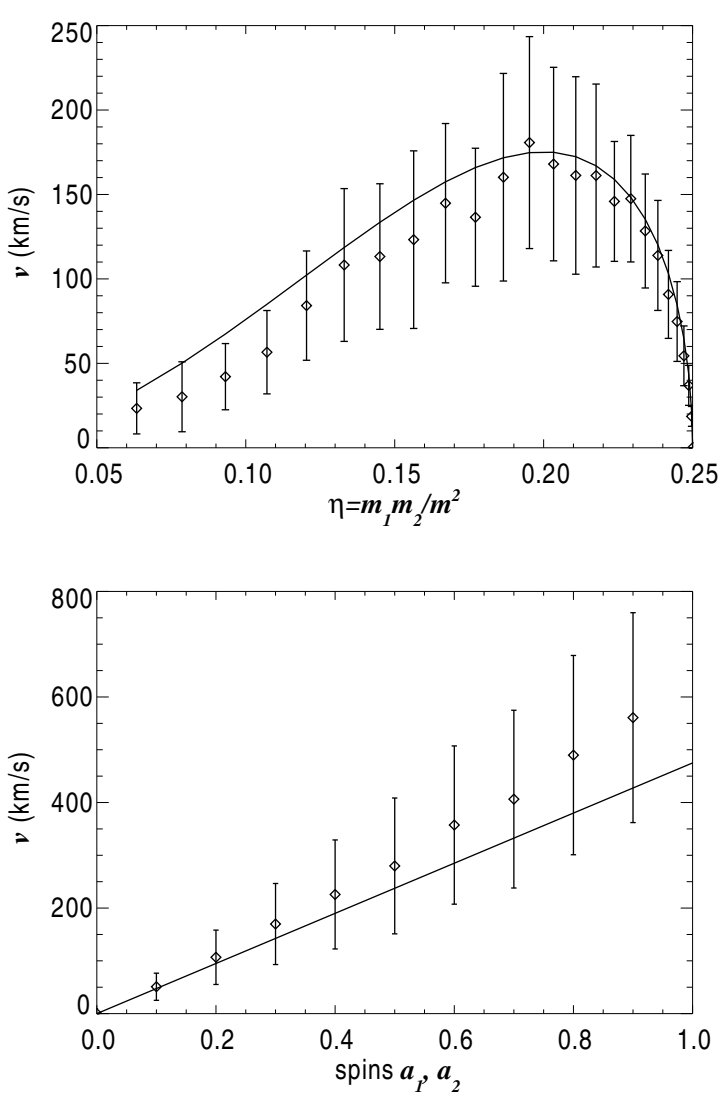

Fig. 1.- Upper: EOB predictions of the recoil velocity for nonspinning unequal-mass BHs, along with 1- $\sigma$ errors. The solid line follows the (Fitchett 1983) scaling, normalized to the NR results in Gonzalez et al. (2006). Lower: Recoil for equal-mass binaries with spins aligned and anti-aligned with the orbital angular momentum. The solid line is the linear fit of Herrmann et al. (2007).

to the EOB predictions of the form

$$
v(q, a)=\frac{32 V_{0} q^{2}}{(1+q)^{5}} \sqrt{(1-q)^{2}+2(1-q) \beta K_{p}+K_{p}^{2}},
$$

where $q=m_{2} / m_{1}$ and $K_{p}=k_{p} a(q+1)$. We agree closely with their best-fit results, matching $V_{0}=276$ $\mathrm{km} / \mathrm{s}$, and finding slightly higher values of $\beta=1.27$, and $k_{p}=1.07$ (compare to their $\beta=0.84$ and $k_{p}=$ 0.85). This deviation is largest for large spins, where they do not have much data and our simplified RD matching methods might start to break down. Following the spin-orbit recoil formula in Kidder (1995), we can modify equation (1) to include non-planar kicks. We write the recoil component in the plane as $K_{p}=$ $k_{p}\left(a_{1} \cos \theta_{1}-q a_{2} \cos \theta_{2}\right)$ and the component out of the plane as $K_{z}=k_{z}\left(a_{1} \sin \theta_{1} \cos \phi_{1}-q a_{2} \sin \theta_{2} \cos \phi_{2}\right)$, with $\theta_{1,2}$ the angle between the spin and angular momentum and $\phi_{1,2}$ the azimuthal angle of each spin, measured with respect to the binary separation vector. From the Monte Carlo simulations (described in the next Section), we find $k_{z} \approx 1.7$, but with a large scatter between the EOB predictions and the simple analytic model.

As a final check of our model, in Table 1 we compare with the more general configurations of Koppitz et al. (2007), Campanelli et al. (2007), and Gonzalez et al. (2007). For the configuration of Gonzalez et al. (2007), 


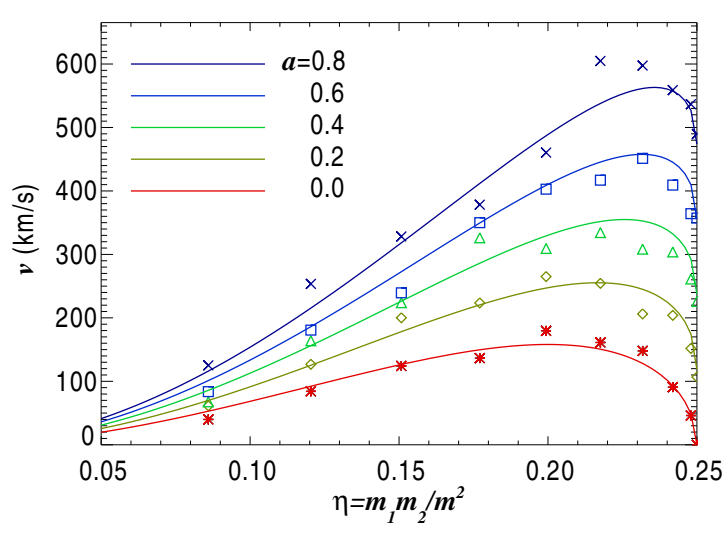

FIG. 2.- EOB predictions of recoil velocity for a range of unequal-mass $\mathrm{BHs}$ with spins aligned and anti-aligned with the orbital angular momentum. The solid lines are fits to equation (1). To avoid confusion, we have not plotted error bars, which are typically $\sim 30-40 \%$ in this case.

TABLE 1

COMPARISON OF NR RECOIL CALCULATIONS WITH EOB MODEL

\begin{tabular}{cllccrrr}
\hline \hline$\frac{m_{1}}{m_{2}}$ & $a_{1}$ & $a_{2}$ & $\begin{array}{c}\theta_{1} \\
(\mathrm{deg})\end{array}$ & $\begin{array}{c}\theta_{2} \\
(\mathrm{deg})\end{array}$ & $\begin{array}{r}v_{\mathrm{NR}} \\
(\mathrm{km} / \mathrm{s})\end{array}$ & $\begin{array}{r}v_{\text {EOB }} \\
(\mathrm{km} / \mathrm{s})\end{array}$ & Ref. \\
\hline 1 & 0.58 & 0.0 & 0 & 180 & $100 \pm 10$ & $120 \pm 70$ & {$[1]$} \\
1 & 0.58 & 0.15 & 0 & 180 & $135 \pm 20$ & $160 \pm 100$ & {$[1]$} \\
1 & 0.58 & 0.29 & 0 & 180 & $185 \pm 5$ & $260 \pm 80$ & {$[1]$} \\
1 & 0.58 & 0.44 & 0 & 180 & $215 \pm 15$ & $420 \pm 210$ & {$[1]$} \\
1 & 0.58 & 0.58 & 0 & 180 & $260 \pm 15$ & $340 \pm 160$ & {$[1]$} \\
2 & 0.8 & 0.0 & 135 & 0 & $454 \pm 25$ & $360 \pm 150$ & {$[2]$} \\
1 & 0.73 & 0.73 & 90 & 90 & $2450 \pm 250$ & $1700 \pm 400$ & {$[3]$} \\
1 & 0.8 & 0.8 & 90 & 90 & $2650 \pm 300$ & $1850 \pm 450$ & {$[3]$} \\
\hline
\end{tabular}

References: [1] Koppitz et al. (2007) [2] Campanelli et al. (2007) [3] Gonzalez et al. (2007)

where the spins are anti-aligned with each other and normal to the angular momentum vector, the value of the final recoil is sensitive to the angle the spins make with the orbital velocity vector. We maximize over this angle, but are still unable to attain their kick magnitudes of $\sim 2500 \mathrm{~km} / \mathrm{s}$. However, we see from these different cases that there doesn't seem to be a systematic disagreement with the NR results: sometimes the EOB overestimates the kick, and sometimes underestimates it, so the velocity distributions integrated over a wide range of $\mathrm{BH}$ parameter space should still be reasonably reliable.

\section{RESULTS: RECOIL VELOCITY DISTRIBUTIONS}

Having successfully compared the EOB model with a range of $\mathrm{NR}$ results, we are now in a position to carry out a series of Monte Carlo calculations. The first model we consider is that of equal-mass BHs with random spins, uniformly distributed in $[0 \leq a \leq 0.9]$ and $[-1 \leq \cos \theta \leq 1]$. We construct a probability distribution function $P(v)$ for the kick velocities by summing over a collection of normal distributions with the mean and variance for each individual binary system calculated as in $\S 2$ The cumulative distribution function $f(v)$ is given by $f(v)=\int_{v}^{\infty} P\left(v^{\prime}\right) d v^{\prime}$, normalized such that $f(0)=1$ and $f(v)$ is the probability that a random binary in the sample will have a recoil larger than $v$.

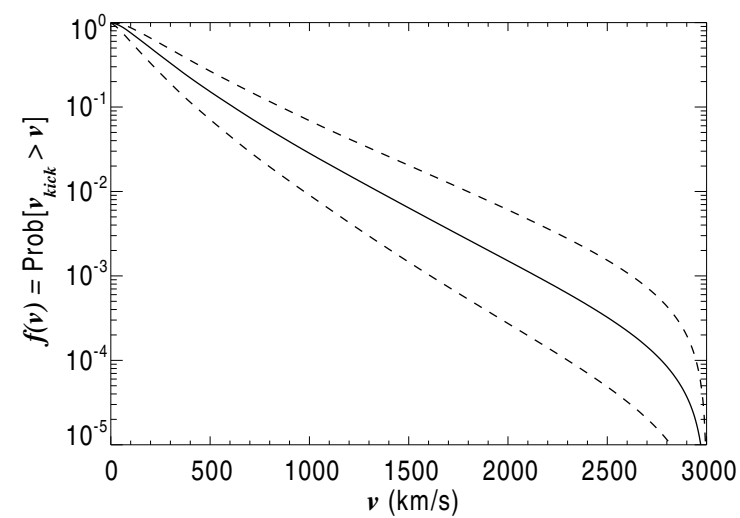

FIG. 3.- Cumulative distribution function for kick velocities $v_{\text {kick }}<v$ for equal masses $m_{1}=m_{2}$ and random spins. The dashed lines represent $1-\sigma$ confidence limits.

Figure 3 shows this distribution for the equal-mass, random spin case for $10^{4}$ binaries, with the dashed lines corresponding to 1- $\sigma$ confidence limits. The resulting uncertainty is on the order of $50 \%$, which, while admittedly large, can still provide interesting new constraints on astrophysical models of black hole mergers.

Perhaps a more realistic model samples a uniform distribution in mass ratios $\left[1 \leq m_{1} / m_{2} \leq 10\right]$ and spin orientations, and set $a=0.9$ for each black hole, based on observational arguments that most supermassive black holes are rapidly spinning (Yu \& Tremaine 2002; Elvis et al. 2002; Wang et al. 2006). For these parameters, we find a fraction $f_{500}=0.12_{-0.05}^{+0.06}$ of binaries have recoil velocities greater than $500 \mathrm{~km} / \mathrm{s}$ and $f_{1000}=0.027_{-0.014}^{+0.021}$ have kicks greater than $1000 \mathrm{~km} / \mathrm{s}$. However, as we saw in $\S 3$, the largest kicks occur with nearly equal masses, so these fractions may be biased towards smaller kicks due to the wide range of $m_{1} / m_{2}$ considered in this model. To factor out the uncertainty in mass distributions, we plot in Figure 4 the velocity distribution function as a function of $\eta$. As expected, the typical velocities increas significantly for $\eta \gtrsim 0.16$. Limiting the sample to this range of masses, we find $f_{500}=0.31_{-0.12}^{+0.13}$ and $f_{1000}=0.079_{-0.042}^{+0.062}$.

It is quite possible that black holes in the early universe have actually gained most of their mass by mergers as opposed to accretion, and thus may not be rapidly spinning (Hughes \& Blandford 2003). To explore this possibility, we performed another calculation with the same range of mass ratios and equal spin magnitudes $a_{1}=a_{2}$, uniformly distributed over $\left[0 \leq a_{1,2} \leq 0.9\right]$, again with random orientations. After calculating the resulting distribution function $f(v ; \eta, a)$, we define the functions $v_{50}(\eta, a)$ as the velocity below which $50 \%$ of the predicted kicks lie. Similarly, $v_{90}(\eta, a)$ is greater than $90 \%$ of the kick velocities for a given $\eta$ and $a$. Contours of these functions are shown in Figure 5. As expected, we see that systems with large spins and nearly equal masses have the highest kicks.

We found by inspection that the distribution function $f(v ; \eta, a)$ can be well approximated for $v \lesssim 1500 \mathrm{~km} / \mathrm{s}$ by

$$
f(v ; \eta, a) \approx \exp \left[-\ln 10\left(\frac{v^{2}}{v_{90}^{2}(\eta, a)}\right)\right] .
$$




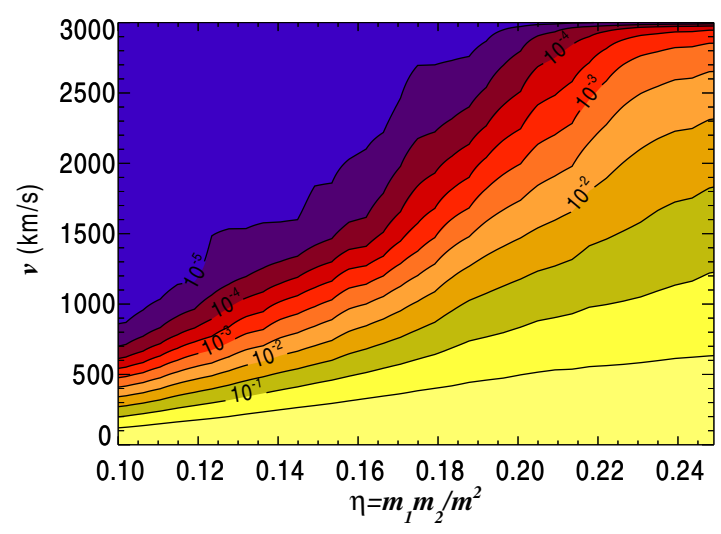

FIG. 4.- Cumulative probability distribution as a function of symmetric mass ratio $\eta$. The contours of $f(v ; \eta)$ represent the probability of having a recoil velocity greater than $v$. The spins have amplitude $a_{1}=a_{2}=0.9$ and random orientation.
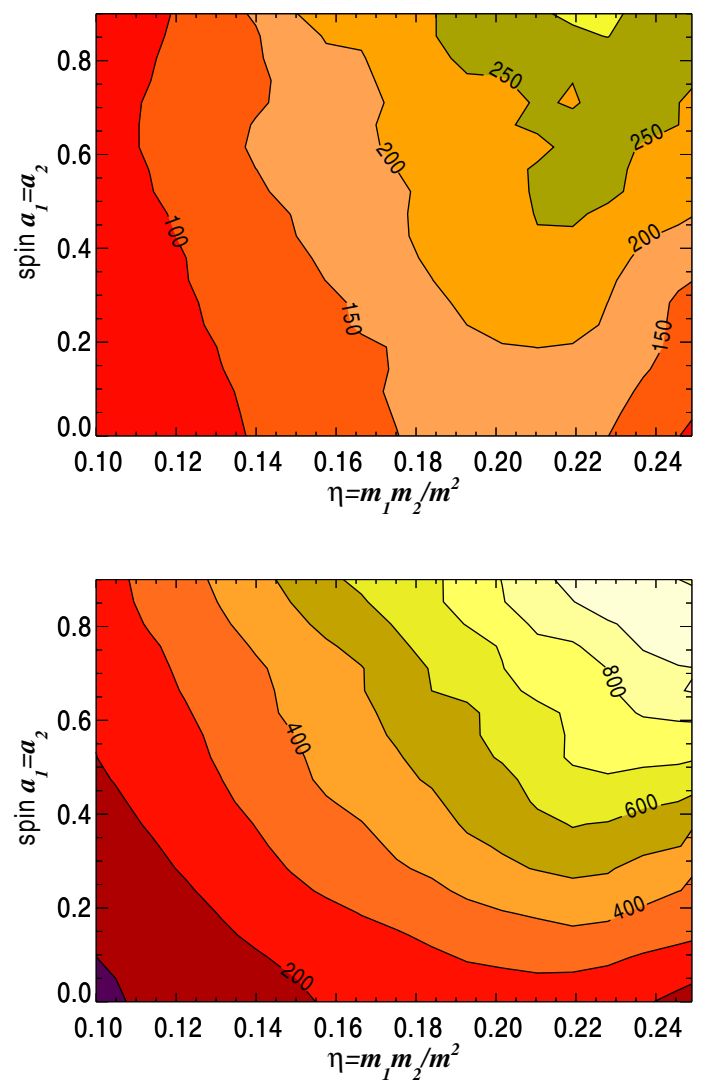

FIG. 5.- Upper: Contours of $v_{50}(\eta, a)$, defined such that $50 \%$ of $\mathrm{BH}$ binaries with a given mass ratio $\eta$ and spin parameter $a$ are expected to have recoil velocities less than $v_{50}$. Lower: Contours of $v_{90}(\eta, a)$, defined analogously to $v_{50}$.
Again motivated by maximizing the phenomenological equation (11), we found a relatively good analytic fit

$$
v_{90}(q, a) \approx \frac{32 V_{0} q^{2}}{(1+q)^{5}} \sqrt{(1-q)^{2}+k^{2} a^{2}(q+1)^{2}}
$$

with $V_{0}=560 \mathrm{~km} / \mathrm{s}$ and $k=1.3$, matching the EOB prediction of $v_{90}$ within $25 \%$ over the entire range of $(\eta, a)$ investigated, and within $10 \%$ for the great majority of it. As a check of this method, we applied it to the other Monte Carlo samples described above and along with equation (2) are able to match closely the integrated distribution functions $f(v)$ for $v \lesssim 1500 \mathrm{~km} / \mathrm{s}$.

\section{DISCUSSION}

Along with the recent NR calculations of recoil velocities, there has been a great deal of discussion of the astrophysical consequences of these kicks. Here we only give a brief summary of these results. For a more detailed review, see Baker et al. (2006); Campanelli et al. (2007), and references therein. Of particular interest to our calculation is the observational evidence that most of the supermassive BHs in the low- $z$ universe are rapidly spinning (Yu \& Tremaine 2002; Elvis et al. 2002; Wang et al. 2006), and may have undergone multiple mergers with $m_{1} / m_{2} \lesssim 3$ (Haehnelt \& Kauffmann 2002; Merritt 2006), and thus have quite possibly received kicks on the order of 500 or even $1000 \mathrm{~km} / \mathrm{s}$. This should be enough to eject the final $\mathrm{BH}$ from spiral galaxy bulges and even most giant elliptical galaxies (Merritt et al. 2004) [globular clusters may favor more extreme mass ratios, but also have significantly smaller escape velocities (Miller \& Hamilton 2002)]. At the same time, there is some observational evidence against such large kicks (Libeskind et al. 2006). This apparent discrepancy may be explained with more detailed models for the BH mass distributions in hierarchical merger scenarios, as well as the possibility of using certain preferred spin orientations that would reduce the kick magnitude (Schnittman 2004). At the same time, to reduce the uncertainties in our predictions, we plan to improve the EOB model, notably the details of the matching method and the determination of the RD frequencies.

A.B. acknowledges support from NSF grant PHY0603762 and from the Alfred Sloan Foundation.

\section{REFERENCES}

Baker, J. G., Centrella, J., Choi, D.-I., Koppitz, M., van Meter, J. R., \& Miller, M. C. 2006 ApJ, 653, L93.

Baker, J. G., Boggs, W. D., Centrella, J., Kelly, B. J., McWilliams, S. T., Miller, M. C., \& van Meter, J. R. 2007, astro-ph/0702390.
Blanchet, L., Damour, T., Esposito-Farese, G., \& Iyer, B. R 2004, Phys. Rev. Lett. 93, 091101.

Blanchet, L., Qusailah, M. S. S., \& Will, C. M. 2005, ApJ 635, 508.

Buonanno, A. \& Damour, T. 1999, Phys. Rev. D 59, 084006. 
Buonanno, A. \& Damour, T. 2000, Phys. Rev. D 62, 064015.

Buonanno, A., Chen, Y. \& Damour, T. 2006, Phys. Rev. D 74, 104005.

Buonanno, A., Cook, G. \& Pretorius, T. 2006, gr-qc/0610122.

Campanelli, M., Lousto, C.O., Zlochower, Y. \& Merritt, D. 2007, gr-qc/0701164.

Damour, 'T., Jaranowski, P., \& Schäfer, G. 2000, Phys. Rev. D 62, 084011.

Damour, T., \& Gopakumar, A. 2006, Phys. Rev. D 73, 124006.

Elvis, M., Risaliti, G., \& Zamorani, G. 2002, ApJ 565, L75.

Fitchett, M. J. 1983, MNRAS 203, 1049.

Flanagan, E. E., \& Hughes, S. A. 1998, Phys. Rev. D 57, 4535.

Gonzalez, J. A., Sperhake, U., Brügmann, B., Hannam, M. D., \& Husa, S. 2006, gr-qc/0610154.

Gonzalez, J. A., Hannam, M. D., M. D., Sperhake, U., Brügmann, B., \& Husa, S. 2007, gr-qc/0702052.

Haehnelt, M. G., \& Kauffmann, G. 2002, MNRAS 336, L61.

Herrmann, F., Shoemaker, D., \& Laguna, P. 2006, gr-qc/0601026.

Herrmann, F., Hinder, I., Shoemaker, D., Laguna, P., \& Matzner, R. A. 2007, gr-qc/0701143.
Hughes, S. A., \& Blandford, R. D. 2003, ApJ 585, L101.

Kidder, L. E. 1995, Phys. Rev. D 52, 821.

Koppitz, M., Pollney, D., Reisswig, C., Rezzolla, L., Thornburg,

J., Diener, P. \& Schnetter, E. 2007, gr-qc/0701163.

Libeskind, N. I., Cole, S., Frenk, C. S., \& Helly, J. C. 2006, MNRAS 368, 1381.

Merritt, D., Milosavljevic, M., Favata, M., \& Hughes, S. A. 2004, ApJ 607, L9.

Merritt, D. 2006, ApJ 648, 976.

Miller, M. C., \& Hamilton, D. P. 2002, MNRAS 330, 232.

Menou, K., Haiman, Z., \& Narayanan, V. K. 2001, ApJ 558, 535.

Sopuerta, C. F., Yunes, N., \& Laguna, P. 2006, astro-ph/0611110.

Schnittman, J. D. 2004, Phys. Rev. D, 124020.

Thorne, K. S. 1980, Rev. Mod. Phys. 52, 299.

Wang, J.-M. 2006, ApJ 642, L111.

Yu, Q. J., \& Tremaine, S. 2002, MNRAS 335, 965. 